\title{
Abundance and composition of zooplankton in the straits of Malacca
}

\begin{abstract}
The zooplankton composition and distribution were studied during four oceanographic cruises in the Straits of Malacca between November 1998 and August 2000 with $140 \mu \mathrm{m}$ mesh and 45-cm diameter NORPAC net. Zooplankton samples were analyzed for the faunal composition and abundance. Zooplankton abundance peaked during pre-southwest monsoon (mean of 12, $918 \pm 5,635$ ind. m3). Copepods were the most important constituents of the zooplankton in all areas. Copepods (including copepodites) accounted for $71.3 \%, 71.2 \%$, $70.9 \%$ and $57.9 \%$ of the total zooplankton populations during consecutive cruises I, II, III and IV, respectively. Molluscs $(6.3 \%$ to $12.4 \%)$ and crustacean's nauplii (4.4\% to $6.0 \%)$ comprised the important groups of non-copepod zooplankton. Spatio-temporal variation in zooplankton abundance was not statistically significant. Zooplankton was more abundant in the near-coastal areas than in offshore areas, but the difference was not significant.
\end{abstract}

Keyword: Copepods; Distribution; Monsoon; Spatio-temporal; Malaysia 